CORRIGENDUM

\title{
Expanding the medicinal chemistry synthetic toolbox
}

Jonas Boström, Dean G. Brown, Robert J. Young and György M. Keserü

Nature Reviews Drug Discovery 17, 709-727 (2018)

The percentages for the five most common reaction types presented in Figure 1a were incorrectly calculated, but the overall message was not affected; these reactions represent the majority $(>60 \%)$ used to produce compounds in drug discovery programmes (J. Med. Chem. 59, 4443-4458; 2015). Figure 1a and its description in the article text have been corrected in the online versions of the article. The authors apologize for the error. 